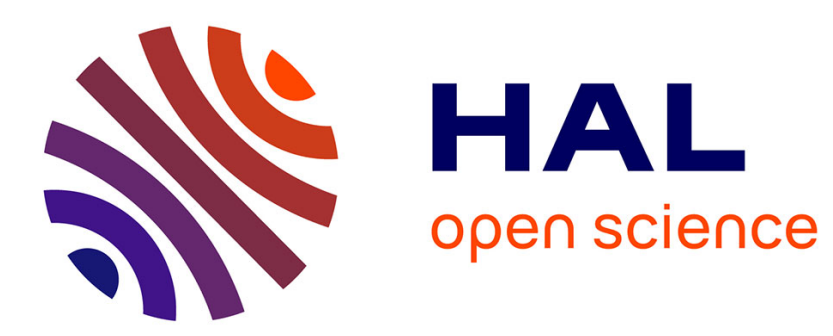

\title{
Subgrid modeling of anisotropic rotating homogeneous turbulence.
}

\author{
L. Shao, Z.S. Zhang, G.X. Cui, C.X. Xu
}

\section{To cite this version:}

L. Shao, Z.S. Zhang, G.X. Cui, C.X. Xu. Subgrid modeling of anisotropic rotating homogeneous turbulence.. Physics of Fluids, 2005, 17, pp.115106. 10.1063/1.2130748 . hal-00022330

\section{HAL Id: hal-00022330 \\ https://hal.science/hal-00022330}

Submitted on 15 Jun 2012

HAL is a multi-disciplinary open access archive for the deposit and dissemination of scientific research documents, whether they are published or not. The documents may come from teaching and research institutions in France or abroad, or from public or private research centers.
L'archive ouverte pluridisciplinaire $\mathbf{H A L}$, est destinée au dépôt et à la diffusion de documents scientifiques de niveau recherche, publiés ou non, émanant des établissements d'enseignement et de recherche français ou étrangers, des laboratoires publics ou privés. 


\title{
Subgrid modeling of anisotropic rotating homogeneous turbulence
}

\author{
L. Shao ${ }^{a)}$ \\ LMFA UMR CNRS 5509, ECL, UCB Lyon I, France
}

Z. S. Zhang, G. X. Cui, ${ }^{\text {b) }}$ and C. X. Xu

Department of Engineering Mechanics, University of Tsinghua, Beijing 100084, China

(Received 4 February 2005; accepted 9 September 2005; published online 28 November 2005)

We investigate subgrid modeling of anisotropic rotating turbulence with a dynamic equation of structure functions of the filtered velocity field. The local volume-averaged structure function equation of rotating turbulence is introduced and an eddy viscosity subgrid model is obtained. The resulting subgrid model is similar to that of the study of Cui et al. [Phys. Fluids 16, 2835 (2004)]. It is directly related to the transfer term: the third-order structure function. This term can be computed dynamically during large eddy simulations (LES). Tests are successfully carried out in LES of decaying, rotating, homogeneous turbulence at high Reynolds numbers. Results are in excellent agreement when compared with those of Cambon et al. [J. Fluid Mech. 337, 303 (1997)]. () 2005 American Institute of Physics. [DOI: 10.1063/1.2130748]

\section{INTRODUCTION}

A new, dynamic subgrid eddy viscosity model $^{1}$ (Cui, Zhou, Zhang, and Shao, hereafter referred to as the "CZZS" model) has recently been derived in which the Kolmogorov equation of the filtered velocity field constitutes the theoretical background. The model has been successfully used in large eddy simulations of homogeneous, isotropic turbulence and channel flow. The Kolmogorov equation of the filtered velocity field is able to provide the right energy transfer between resolved and unresolved scales. Based on this previous work, ${ }^{1}$ we now extend the study to anisotropic rotating turbulence. The main effects of rotating turbulence are (1) the spectral energy transfer is reduced and (2) the turbulence is anisotropic. The higher the rotation rate, the more pronounced the anisotropy and reduction of energy transfer. The kinetic energy spectrum is believed to behave as $K^{-3}$ for high rotation rates (Yang and Domaradzki ${ }^{2}$ ), with $K$ being the wave number. For an extensive review of rotating turbulence, the reader is referred to a recent paper of Cambon et al. ${ }^{3}$ To our knowledge, in the framework of subgrid modeling, there are few formal anisotropic formulations of subgrid eddy viscosity (see Cottet ${ }^{4,5}$ ). In this study, we use an anisotropic formulation of the structure function equations for rotating turbulence, and derive an eddy viscosity which includes the anisotropy in the energy transfer between resolved and unresolved scales. This model is then tested in rotating and decaying homogeneous turbulence at high Reynolds numbers. Results are compared with those of Cambon et $a l .{ }^{6}$ Particular attention is paid to the spectral transfer reduction versus micro-Rossby number and to the spectral slope of the kinetic energy spectrum, since these quantitative comparisons are available.

\footnotetext{
${ }^{a)}$ Electronic mail: shao@mecaflu.ec-lyon.fr

${ }^{b)}$ Electronic mail: cgx@mail.tsinghua.edu.cn
}

\section{FORMULATION OF THE SUBGRID EDDY VISCOSITY FOR ROTATING TURBULENCE}

The energy cascade of isotropic turbulence is well described by the classic Kolmogorov equation. The Kolmogorov equation of the filtered velocity field represents the correct energy transfer between resolved and unresolved scales (CZZS, Ref. 1). However, both classic and modified Kolmogorov equations are only valid in isotropic turbulence. Recently, Hill ${ }^{7}$ and Casciola et al. $^{8}$ derived a volumeaveraged Kolmogorov equation which is valid in both isotropic and anisotropic turbulence. We have since derived the volume-averaged Kolmogorov equation for filtered velocity fields, and propose a new subgrid eddy viscosity model for anisotropic and rotating turbulence.

The governing equation of LES for homogeneous rotating turbulence can be written as

$$
\begin{aligned}
& \frac{\partial \bar{u}_{i}}{\partial t}+\bar{u}_{k} \frac{\partial \bar{u}_{i}}{\partial x_{k}}=-\frac{\partial \bar{p}}{\partial x_{i}}+\nu \frac{\partial^{2} \bar{u}_{i}}{\partial x_{k} \partial x_{k}}+\epsilon_{i k 3} \Omega \bar{u}_{k}+\frac{\partial \tau_{i k}}{\partial x_{k}}, \\
& \frac{\partial \bar{u}_{i}}{\partial x_{i}}=0,
\end{aligned}
$$

in which $\Omega$ is the rotation rate in the $x_{3}$ direction, and $\tau_{i j}$ $=\bar{u}_{i} \bar{u}_{j}-{\overline{u_{i}}}_{j}$ is the subgrid stress. To derive the dynamic equation for structure functions, i.e., the equation for the covariance of a velocity increment, $\Delta \bar{u}_{i}=\bar{u}_{i}(t, \mathbf{x})-\bar{u}_{i}(t, \mathbf{x}-\mathbf{r})$, the following equation is needed for $\bar{u}_{i}(t, \mathbf{x}-\mathbf{r})$ :

$$
\begin{aligned}
& \frac{\partial \bar{u}_{i}^{\prime}}{\partial t}+\bar{u}_{k}^{\prime} \frac{\partial \bar{u}_{i}^{\prime}}{\partial x_{k}^{\prime}}=-\frac{\partial \bar{p}^{\prime}}{\partial x_{i}^{\prime}}+\nu \frac{\partial^{2} \bar{u}_{i}^{\prime}}{\partial x_{k}^{\prime} \partial x_{k}^{\prime}}+\epsilon_{i k 3} \Omega \bar{u}_{k}^{\prime}+\frac{\partial \tau_{i k}^{\prime}}{\partial x_{k}^{\prime}}, \\
& \frac{\partial \bar{u}_{i}^{\prime}}{\partial x_{i}^{\prime}}=0,
\end{aligned}
$$

in which the superscript ${ }^{\prime}$ denotes quantities at point $\mathbf{x}^{\prime}=\mathbf{x}$ $-\mathbf{r}$. 
Subtracting Eq. (1) from Eq. (3), and keeping in mind that $\partial \bar{u}_{i}^{\prime} / \partial x_{i}=0$ and $\partial \bar{u}_{i} / \partial x_{i}^{\prime}=0$, we get

$$
\begin{aligned}
\frac{\partial \Delta \bar{u}_{i}}{\partial t} & +\bar{u}_{k} \frac{\partial \Delta \bar{u}_{i}}{\partial x_{k}}+\bar{u}_{k}^{\prime} \frac{\partial \Delta \bar{u}_{i}}{\partial x_{k}^{\prime}} \\
= & -\frac{\partial \bar{p}}{\partial x_{i}}+\frac{\partial \bar{p}^{\prime}}{\partial x_{i}}+\nu \frac{\partial^{2} \Delta \bar{u}_{i}}{\partial x_{k} \partial x_{k}}+\nu \frac{\partial^{2} \Delta \bar{u}_{i}}{\partial x_{k}^{\prime} \partial x_{k}^{\prime}}+\epsilon_{i k 3} \Omega \Delta \bar{u}_{k} \\
& +\frac{\partial \tau_{i k}}{\partial x_{k}}-\frac{\partial \tau_{i k}^{\prime}}{\partial x_{k}^{\prime}} .
\end{aligned}
$$

Multiplying Eq. (5) by $\Delta \bar{u}_{i}$, we find

$$
\begin{aligned}
& \frac{\partial \Delta \bar{u}_{i}^{2} / 2}{\partial t}+\bar{u}_{k} \frac{\partial \Delta \bar{u}_{i}^{2} / 2}{\partial x_{k}}+\bar{u}_{k}^{\prime} \frac{\partial \Delta \bar{u}_{i}^{2} / 2}{\partial x_{k}^{\prime}} \\
& =-\Delta \bar{u}_{i} \frac{\partial \bar{p}}{\partial x_{i}}+\Delta \bar{u}_{i} \frac{\partial \bar{p}^{\prime}}{\partial x_{i}}+\nu \frac{\partial^{2} \Delta \bar{u}_{i}^{2} / 2}{\partial x_{k} \partial x_{k}}+\nu \frac{\partial^{2} \Delta \bar{u}_{i}^{2} / 2}{\partial x_{k}^{\prime} \partial x_{k}^{\prime}} \\
& -\nu \frac{\partial \Delta \bar{u}_{i}}{\partial x_{k}} \frac{\partial \Delta \bar{u}_{i}}{\partial x_{k}}-\nu \frac{\partial \Delta \bar{u}_{i}}{\partial x_{k}^{\prime}} \frac{\partial \Delta \bar{u}_{i}}{\partial x_{k}^{\prime}}+\Delta \bar{u}_{i} \frac{\partial \tau_{i k}}{\partial x_{k}}-\Delta \bar{u}_{i} \frac{\partial \tau_{i k}^{\prime}}{\partial x_{k}^{\prime}} \\
& +\epsilon_{i k 3} \Omega \Delta \bar{u}_{k} \Delta \bar{u}_{i} .
\end{aligned}
$$

Note that the final term of the last equation equals zero, since $\epsilon_{i k 3}=-\epsilon_{k i 3}$. This means that the rotation effect is implicitly involved in the energy transfer. Similarly, in spectral space, the effect of rotation is also implicitly included in the transfer term. The rotation cannot change the total turbulent energy, but can transfer kinetic energy among different velocity components and make the turbulence anisotropic. Taking the ensemble average of Eq. (6), note that $\partial / \partial x_{i}=\partial / \partial r_{i}, \partial / \partial x_{i}^{\prime}$ $=-\partial / \partial r_{i}, \partial\left\langle p u_{i}\right\rangle / \partial x_{i}=0$, and $\partial\left\langle p^{\prime} u_{i}^{\prime}\right\rangle / \partial x_{i}^{\prime}=0$. In homogeneous turbulence, the governing equation for the second order structure function for rotating turbulence is

$$
\begin{aligned}
\frac{\partial \bar{D}_{i i}}{\partial t}+\frac{\partial \bar{D}_{i i k}}{\partial r_{k}}= & 2 \nu \frac{\partial^{2} \bar{D}_{i i}}{\partial r_{k} \partial r_{k}}-4 \bar{\epsilon}+2\left\langle\Delta \bar{u}_{i} \frac{\partial \tau_{i k}}{\partial x_{k}}\right\rangle \\
& -2\left\langle\Delta \bar{u}_{i} \frac{\partial \tau_{i k}^{\prime}}{\partial x_{k}^{\prime}}\right\rangle .
\end{aligned}
$$

The continuity equation is used, i.e., $\partial \Delta \bar{u}_{i} / \partial x_{i}=\partial \Delta \bar{u}_{i} / \partial x_{i}^{\prime}=0$, in the derivation of the above equation. In Eq. (7), $\bar{D}_{i i}$ $=\left\langle\Delta \bar{u}_{i} \Delta \bar{u}_{i}\right\rangle$ and $\bar{D}_{i i k}=\left\langle\Delta \bar{u}_{i} \Delta \bar{u}_{i} \Delta \bar{u}_{k}\right\rangle$ are second- and third-order structure functions, respectively, and $\bar{\epsilon}=\nu\left\langle\partial \bar{u}_{i} / \partial x_{k} \partial \bar{u}_{i} / \partial x_{k}\right\rangle$ is the molecular dissipation of resolved scale turbulence. For unfiltered isotropic turbulence, Eq. (7) can be simplified to the classic Kolmogorov equation by use of tensor rules; however, in anisotropic turbulence, such as rotating turbulence, both $\bar{D}_{i i}$ and $\bar{D}_{i i k}$ are functions of the vector $\mathbf{r}$. We show that the last two terms can be further simplified when an eddy viscosity is assumed for the subgrid stress tensor $\tau_{i j}$

$$
\tau_{i j}=-2 \nu_{t} \bar{S}_{i j}+\tau_{k k} \delta_{i j} / 3
$$

In the above expression, $\bar{S}_{i j}$ is the deformation tensor of the resolved scales. Subgrid eddy viscosity is assumed to be spatially constant for homogeneous turbulence. This eddy viscosity may be subsequently time dependent for unsteady flow.
The last two terms of Eq. (7) can be expressed as

$$
\begin{aligned}
T_{i i} & =2\left\langle\Delta \bar{u}_{i} \frac{\partial \tau_{i k}}{\partial x_{k}}\right\rangle-2\left\langle\Delta \bar{u}_{i} \frac{\partial \tau_{i k}^{\prime}}{\partial x_{k}^{\prime}}\right\rangle \\
& =2\left\langle\Delta \bar{u}_{i}\left(\frac{\partial \tau_{i k}}{\partial x_{k}}-\frac{\partial \tau_{i k}^{\prime}}{\partial x_{k}^{\prime}}\right)\right\rangle \\
& =2\left\langle\frac{\partial\left(\Delta \bar{u}_{i} \tau_{i k}\right)}{\partial x_{k}}-\frac{\partial\left(\Delta \bar{u}_{i} \tau_{i k}^{\prime}\right)}{\partial x_{k}^{\prime}}\right\rangle-2\left\langle\tau_{i k} \frac{\partial \Delta \bar{u}_{i}}{\partial x_{k}}\right. \\
& \left.-\tau_{i k}^{\prime} \frac{\partial \Delta \bar{u}_{i}}{\partial x_{k}^{\prime}}\right\rangle .
\end{aligned}
$$

Note that the isotropic part of the subgrid stress does not contribute to $T_{i i}$ in homogeneous turbulence for the same reason as pressure does not contribute to the energy transfer in homogeneous turbulence. We insert the subgrid stress into the above equation to obtain the final expression of $T_{i i}$ as

$$
T_{i i}=2 \nu_{t} \frac{\partial^{2} \bar{D}_{i i}}{\partial r_{k} \partial r_{k}}+4 \nu_{t}\left\langle\frac{\partial \bar{u}_{i}}{\partial x_{k}} \frac{\partial \bar{u}_{i}}{\partial x_{k}}\right\rangle \text {. }
$$

Inserting Eq. (10) into Eq. (7), we have

$$
\frac{\partial \bar{D}_{i i}}{\partial t}+\frac{\partial \bar{D}_{i i k}}{\partial r_{k}}=2\left(\nu+\nu_{t}\right) \frac{\partial^{2} \bar{D}_{i i}}{\partial r_{k} \partial r_{k}}+4\left(\nu+\nu_{t}\right)\left\langle\frac{\partial \bar{u}_{i}}{\partial x_{k}} \frac{\partial \bar{u}_{i}}{\partial x_{k}}\right\rangle \text {. }
$$

Following $\mathrm{Hill}^{7}$ and Casciola et al., ${ }^{8}$ we take volume integration over a sphere with radius $r$

$$
\begin{aligned}
& \int_{V}\left\{\frac{\partial \bar{D}_{i i}}{\partial t}+\frac{\partial \bar{D}_{i i k}}{\partial r_{k}}\right\} d v \\
& =\int_{v}\left\{2\left(\nu+\nu_{t}\right) \frac{\partial^{2} \bar{D}_{i i}}{\partial r_{k} \partial r_{k}}+4\left(\nu+\nu_{t}\right)\left\langle\frac{\partial \bar{u}_{i}}{\partial x_{k}} \frac{\partial \bar{u}_{i}}{\partial x_{k}}\right\rangle\right\} d v .
\end{aligned}
$$

In virtue of the Green-Ostrograsky integration formula, the volume integration can be transferred to the surface integration

$$
\int_{V} \frac{\partial \bar{D}_{i i k}}{\partial r_{k}} d v=\oint_{S_{r}} D_{i i k} n_{k} d A=\oint_{S_{r}} D_{i i r} d A(r)
$$

and

$$
\int_{V} \frac{\partial^{2} \bar{D}_{i i}}{\partial r_{k} \partial r_{k}} d v=\oint_{S_{r}} \frac{\partial \bar{D}_{i i}}{\partial r_{k}} n_{k} d A=\oint_{S_{r}} \frac{\partial \bar{D}_{i i}}{\partial r} d A(r),
$$

where $d A(r)$ is the element surface of the sphere. Inserting the above two identities into Eq. (12), we have

$$
\begin{aligned}
\frac{\partial \bar{D}_{i i}^{V}}{\partial t}+\frac{S_{r}}{V} \bar{D}_{i i r}^{S_{r}}= & \frac{2\left(\nu+\nu_{t}\right) S_{r}}{V}\left(\frac{\partial \bar{D}_{i i}}{\partial r}\right)^{S_{r}}-4\left(\nu+\nu_{t}\right) \\
& \times\left\langle\frac{\partial \bar{u}_{i}}{\partial x_{k}} \frac{\partial \bar{u}_{i}}{\partial x_{k}}\right\rangle^{V},
\end{aligned}
$$

in which the superscript $V$ and $S_{r}$ denote the volume and surface average, respectively, e.g., $\bar{D}_{i i}^{V}=1 / V \int_{V} \bar{D}_{i i} d v$ and $\bar{D}_{i i}^{S_{r}}$ 


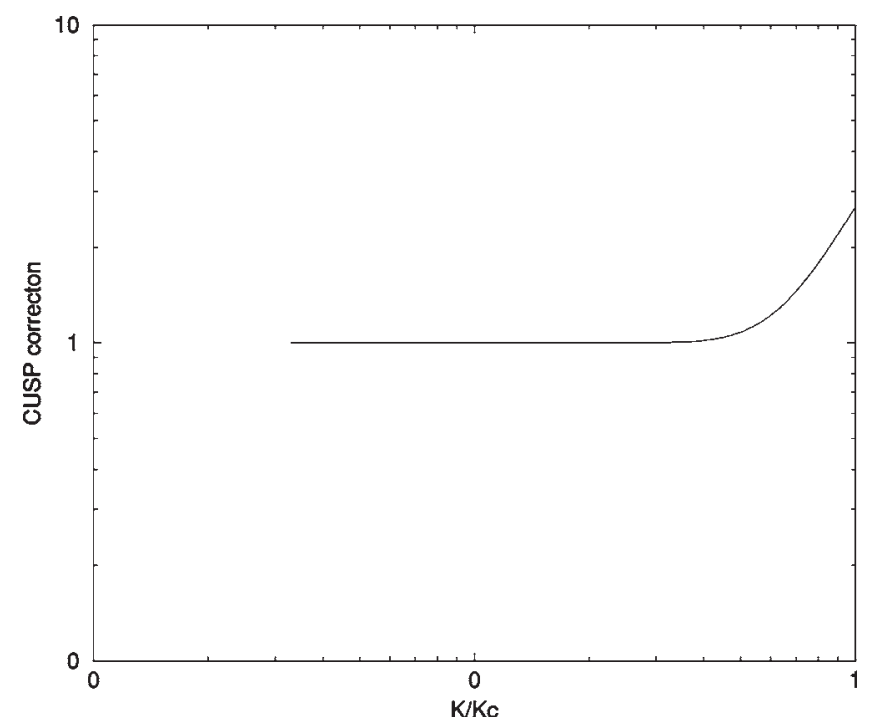

FIG. 1. CUSP effect correction of Chollet (Ref. 9) in a $96 \times 384^{*} 394$ LES run.

$=1 / S_{r} \oint_{S_{r}} \bar{D}_{i i} d A(r)$. For a sphere, $S_{r} / V=3 / r$. The volumeaveraged equation can be written as

$$
\frac{\partial \bar{D}_{i i}^{V}}{\partial t}+\frac{3}{r} \bar{D}_{i i r}^{S_{r}}=\frac{6\left(\nu+\nu_{t}\right)}{r}\left(\frac{\partial \bar{D}_{i i}}{\partial r}\right)^{S_{r}}-4\left(\nu+\nu_{t}\right)\left\langle\frac{\partial \bar{u}_{i}}{\partial x_{k}} \frac{\partial \bar{u}_{i}}{\partial x_{k}}\right\rangle^{V} .
$$

Neglecting molecular viscosity for high Reynolds number turbulence, and dropping the time derivative term for small $r$, we have the subgrid eddy viscosity for anisotropic turbulence

$$
\nu_{t}=\frac{3 \overline{D_{i i r}}{ }^{s}}{6 \frac{\partial D_{i i} s}{\partial r}-4\left\langle\frac{\partial \bar{u}_{i}}{\partial x_{k}} \frac{\partial \bar{u}_{i}}{\partial x_{k}}\right\rangle} .
$$

\section{NUMERICAL APPLICATIONS: LES OF ROTATING DECAYING HOMOGENEOUS TURBULENCE}

\section{A. Numerical method}

The numerical method used is a classic pseudospectral method. Due to the particular spectral cutoff filter in this numerical method, there is an increasing effect of the truncation of triadic interaction near the cutoff wave number, namely the CUSP effect. Since our subgrid eddy viscosity is assumed to be spatially constant and wave-number independent, the CUSP effect, in addition to the subgrid eddy viscosity, is accounted for by a correction from Chollet ${ }^{9}$ illustrated in Fig. 1. This correction leads to an increase of the subgrid dissipation of about $30 \%$. In order to simulate infinite Reynolds number turbulence, the molecular viscosity is set to zero. The initial field is generated using the method of Rogallo $^{10}$ with a von Kármán spectrum. The numerical domain is a rectangular box. In the direction of rotation, the box length is 4 times larger. As rotation is present, the length scale growth is greater in this direction. The fourth-order
Runge-Kutta method is used for time advance. The time step is set to be sufficiently small to allow the inertial wave resolution.

\section{B. Time relaxation}

The eddy viscosity is directly connected to the thirdorder structure functions. Third-order structure functions may vary rapidly in time while turbulent dissipation is slow. The third-order term is connected to a convection time at filter size, and the subgrid viscous term is connected to the time variation of second-order structure functions. As the aim of the present paper is to simulate high Reynolds decaying rotating turbulence, a time relaxation is used.

Subgrid eddy viscosity is relaxed over a time period $T_{M}$. At time step $n+1, \nu_{t}^{n+1}$ is computed as follows:

$$
\nu_{t}^{n+1}=\left(1-\frac{\Delta t}{T_{M}}\right) \nu_{t}^{n}+\frac{\Delta t}{T_{M}} \nu_{t}^{n+1},
$$

where $\Delta t$ is the time step.

The time period $T_{M}$ is assumed to be the time variation of the second-order structure function at filter size $\Delta . T_{M}$ can be evaluated with Eq. (7) at the filter size $r=\Delta$ :

$$
\begin{aligned}
& {\left[\bar{D}_{i i}\right]=u^{\prime 2},} \\
& {\left[\bar{D}_{i i k}\right]=u^{\prime 3},} \\
& {\left[\nu_{t}\right]=\frac{\Delta^{2}}{T_{\mathrm{vis}}},} \\
& {\left[\frac{\partial \bar{D}_{i i}}{\partial t}\right]=\frac{u^{\prime 2}}{T_{M}},} \\
& {\left[\frac{\partial \bar{D}_{i i k}}{\partial r}\right]=\frac{u^{\prime 3}}{\Delta},}
\end{aligned}
$$$$
\text { [viscous term] }=\frac{\Delta^{2}}{T_{\text {vis }}} \frac{u^{\prime 2}}{\Delta^{2}} .
$$

$T_{M}$ is then estimated by

$$
\frac{1}{T_{M}}=\frac{u^{\prime}}{\Delta}+\frac{1}{T_{\text {vis }}}
$$

where $T_{\text {vis }}$ is evaluated during simulation with the instantaneous eddy viscosity. At $t=0$, the value of the subgrid eddy viscosity is initiated by the value of the asymptotic isotropic skewness model (AISM model) [expression (5.5) in Cui et $\left.a l^{1}{ }^{1}\right]$, and the value of the skewness was set to -0.49 .

\section{Decaying high Reynolds turbulence}

At first the validity of the model equation (17) is assessed in decaying isotropic turbulence. A $64^{3}$ cubic numerical grid is used. Figure 2 shows the time evolution of resolved scale turbulent kinetic energy. The classic power law, $t^{-n}$, with $n \approx 1.25$, is obtained in a short time. In order to check the long-time behavior of the present subgrid eddy viscosity, the simulation is run for more than $10^{6}$ initial turn- 


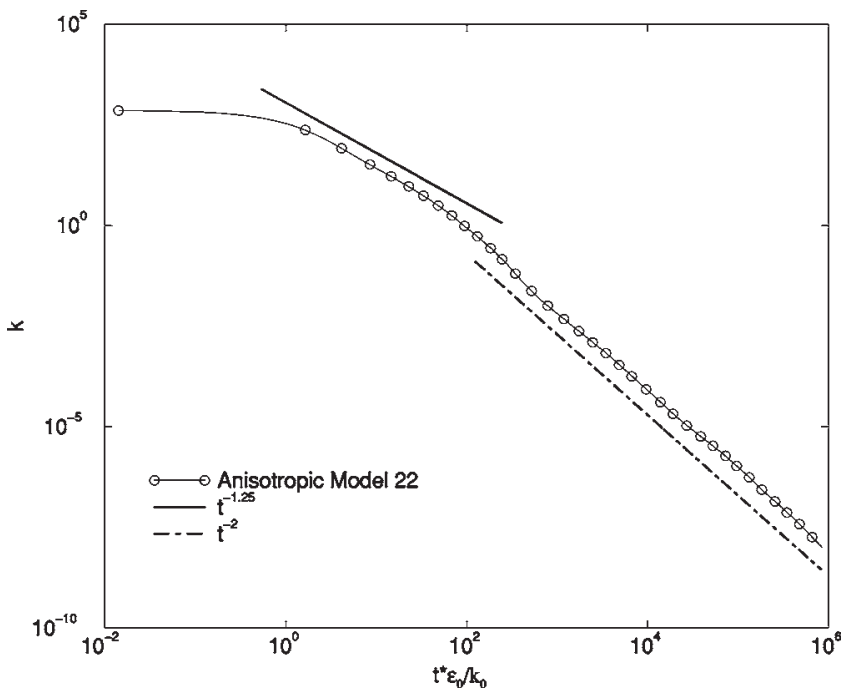

FIG. 2. Time variation of large-scale kinetic energy in a $64^{3}$ LES run.

over times. The effect of the integral length-scale growth blocked by the numerical box due to the "infrared cutoff" wave number is studied in detail by Touil et al. ${ }^{11}$ In the present simulation, this effect is correctly recovered. As an example, at the final decay stage, the power law is shifted to $t^{-2}$. This result is also consistent with the experimental study of Skrbek and Stalp. ${ }^{12}$ Moreover, as shown in Fig. 3, after the length-scale saturation, nondimensional spectra $E(K) \epsilon_{f}^{-2 / 3} K^{5 / 3}$ exhibit the clear range of a Kolmogorov spectrum. The averaged Kolmogorov constant over the plateau is 1.8. We conclude that the present model is able to perform high Reynolds homogeneous turbulence simulations.

\section{Rotating turbulence results}

For the large eddy simulation of a decaying rotating high Reynolds homogeneous turbulence, a precomputation of purely decaying isotropic turbulence is performed. The pre-

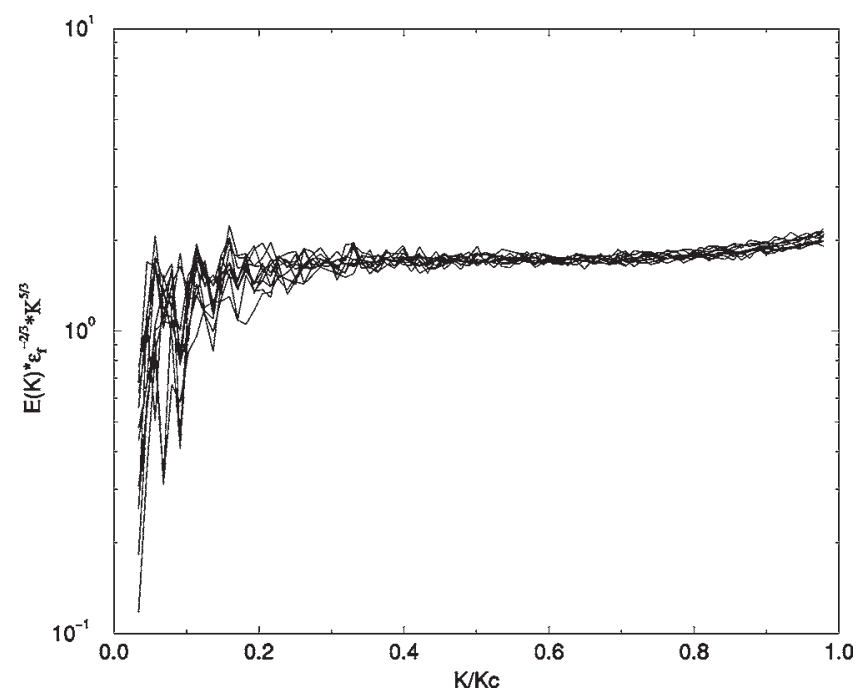

FIG. 3. Spectral behavior after the length-scale saturation, at different times in a $64^{3}$ LES run.

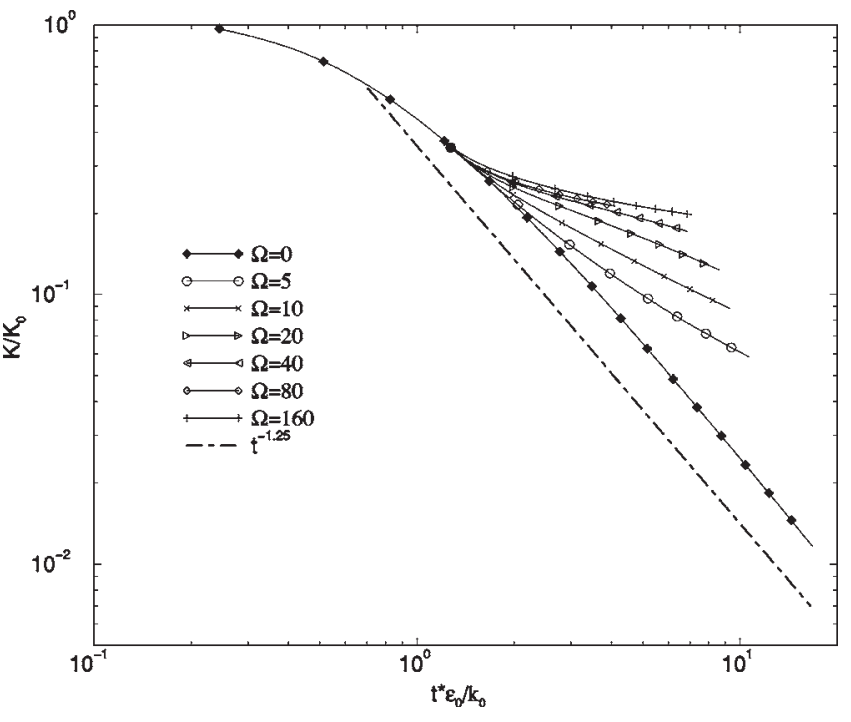

FIG. 4. Time variation of large-scale kinetic energy, for different rotation rates in a $64 \times 64 \times 256$ LES run.

computation is run until the time decay of large-scale kinetic energy $k$ reaches a reliable power law; solid body rotation is then switched on.

Figure 4 shows the time variation of the large-scale kinetic energy for different rotation rates. The numerical grid is a $64 \times 64 \times 256$ rectangular box. As the rotation reduces the spectral energy transfer, the decay of the turbulent kinetic energy is slower. This phenomenon is more pronounced when the rotation rate increases. The time variation of subgrid eddy viscosity is also plotted in Fig. 5. Subgrid viscosity is initially reduced when rotation is switched on. It then decays slower than in the nonrotation case. At the end of computation, the level of the subgrid viscosity is larger than in the case of rotating turbulence. This is due to the fact that the kinetic energy decays much faster in nonrotating turbulence, and that the level of subgrid eddy viscosity is related to the subgrid energy. To quantify these reductions, the ratio of the

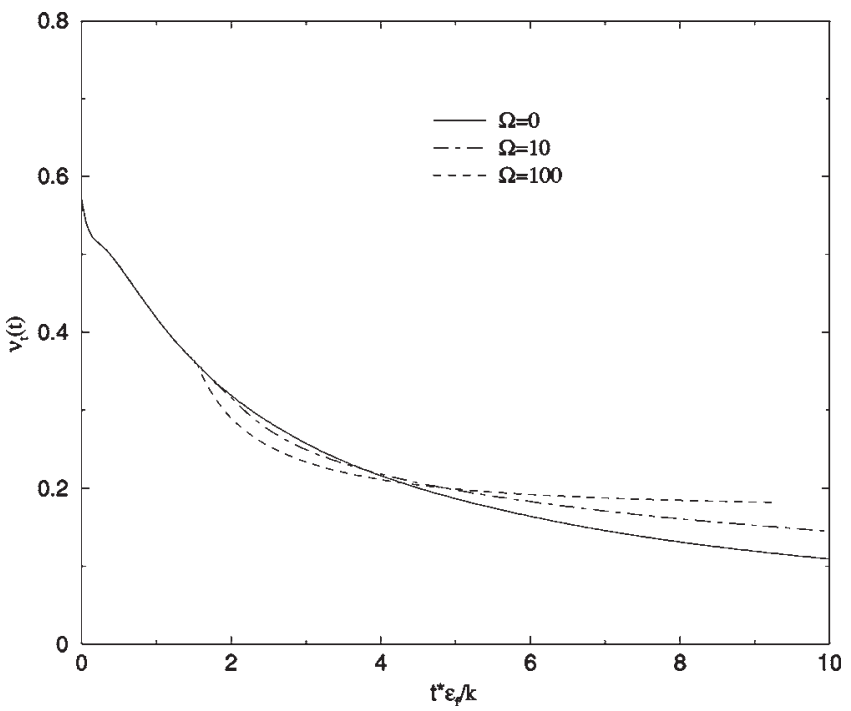

FIG. 5. Time variation of subgrid eddy viscosity, for different rotation rates in a $64 \times 64 \times 256$ LES run. 


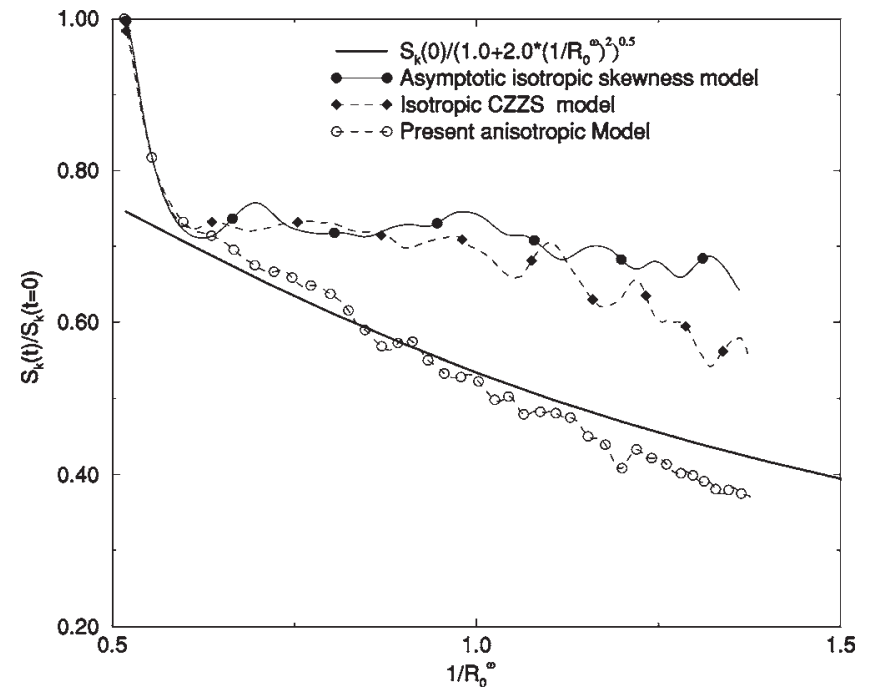

FIG. 6. Skewness variation vs micro-Rossby number with rotation rate $\Omega$ $=10$. A comparison between AISM, isotropic CZZS, and the present model in a $64 \times 64 \times 256$ LES run.

derivative skewness $S_{k} / S_{k}$ (at $\Omega=0$ ) versus the rotation rate is checked. The derivative skewness quantifies the mechanisms of energy transfer and energy dissipation. It is usually related to the small-scale turbulence. In LES, small scales are not present. The derivative skewness represents the mechanisms of energy transfer and subgrid dissipation. In both cases, the derivative skewness is a balance between energy transfer and dissipation mechanisms. A good LES should be able to correctly reproduce the modification of this balance by rotation. Although a direct comparison of the value of the derivative skewness between LES and direct numeric simulation is meaningless, comparison of its variation under the influence of rotation can indicate the ability of the selfadjustment of a subgrid model to the rotation effect.

In a decaying turbulence, when rotation is applied, the skewness is reduced with time while the Rossby number

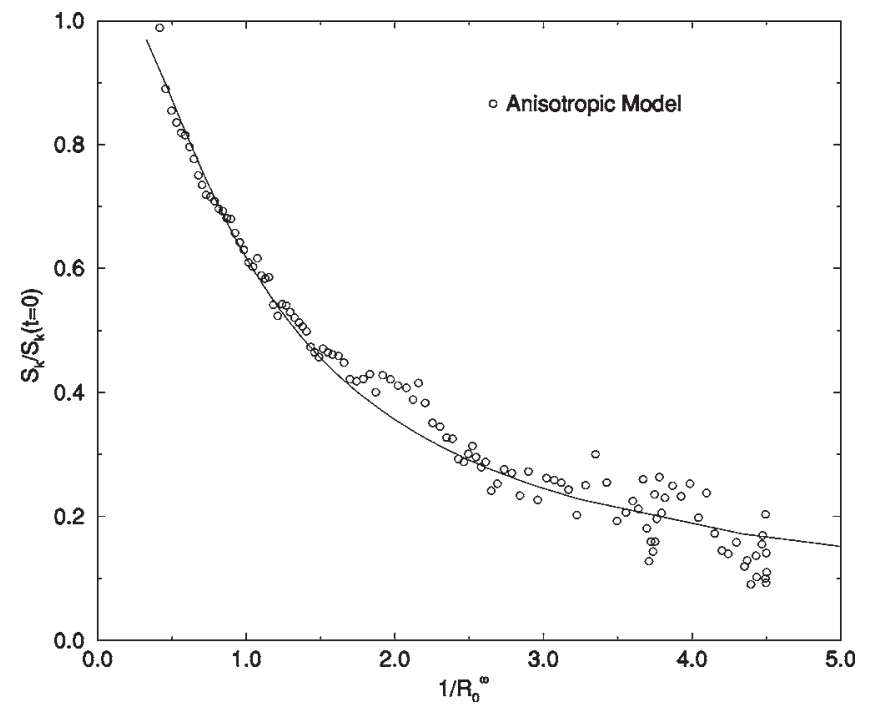

FIG. 7. Skewness variation vs micro-Rossby number with a rotation rate of $\Omega=10$. The solid line represents the Cambon et al. (Ref. 6) model, and circles the present model in a $96 \times 96 \times 384$ LES run.

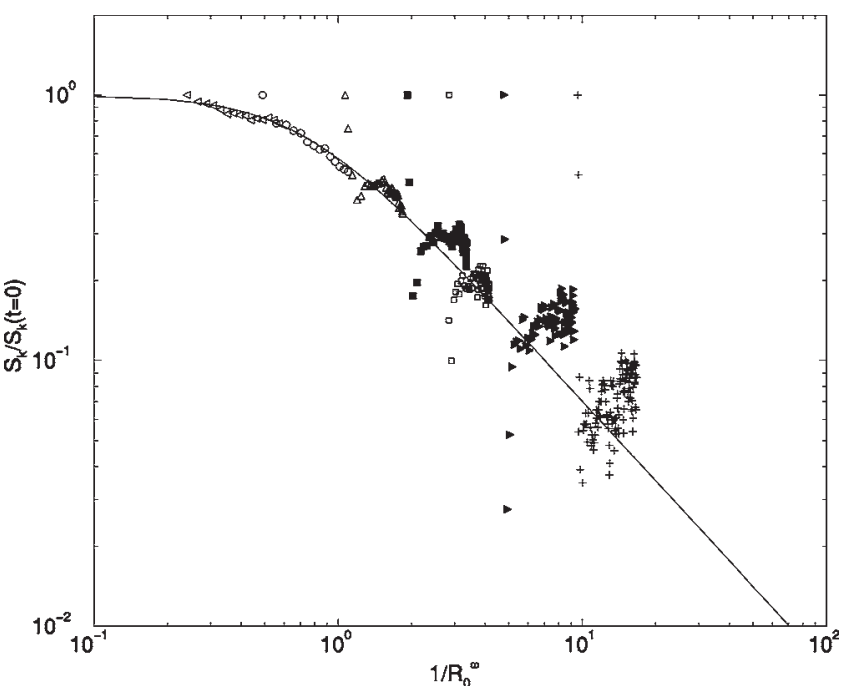

FIG. 8. Skewness variation vs micro-Rossby number with different initial rotation rates. The solid line represents the Cambon et al. (Ref. 6) model, and symbols the present model in a $64 \times 64 \times 256$ LES run.

increases. Cambon et al. ${ }^{6}$ propose a scaling law for the skewness reduction. The derivative skewness $S_{k}$ versus the microRossby number $R_{o}^{\omega}$ is linked by the following relationship:

$$
\frac{S_{k}}{S_{k}(t=0)}=\frac{1.0}{\left(1+2 R_{o}^{\omega-2}\right)^{0.5}},
$$

where $S_{k}(t=0)$ is the value reached by the nonrotation precomputation, and $R_{o}^{\omega}$ is evaluated by $(\epsilon / k \Omega)\left(k^{2} / \nu \epsilon\right)^{0.5}$. The turbulent dissipation $\epsilon$ is replaced by $\epsilon_{f}$, the molecular viscosity $\nu$ by $\nu_{t}$, and the large-scale kinetic energy $k$ is used in LES.

For comparison, the AISM model ${ }^{1}$ and the isotropic $\mathrm{CZZS} \mathrm{model}^{1}$ are also used. Computations are performed on a $64 \times 64 \times 256$ numerical grid. Figure 6 shows the time variation of the velocity derivative skewness versus the inverse of the instantaneous micro-Rossby number. The rotation rate is $\Omega=10$, corresponding to an initial micro-Rossby

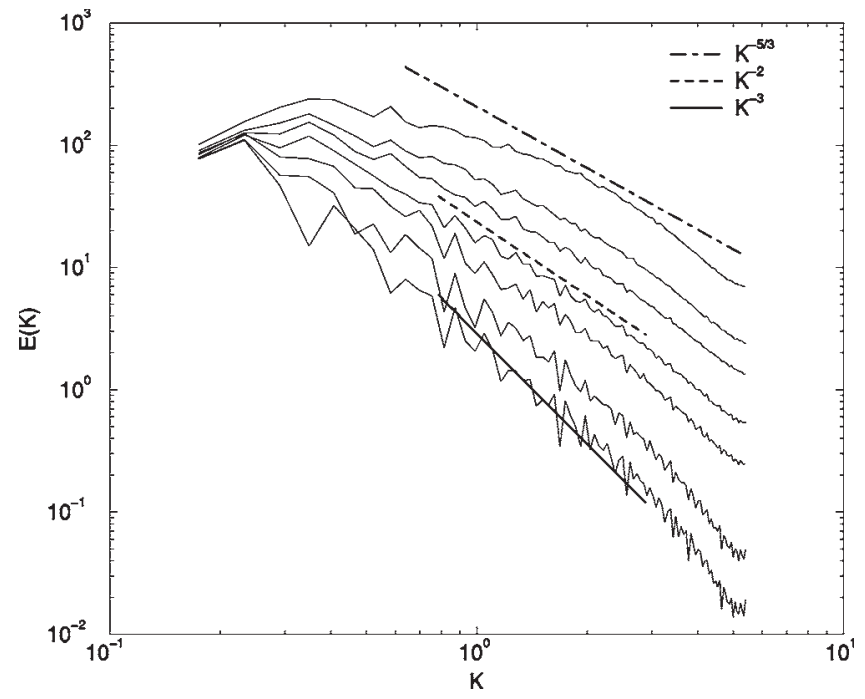

FIG. 9. Time variation of spectrum with a rotation rate of $\Omega=10$ in a 96 $\times 96 \times 384$ LES run. 


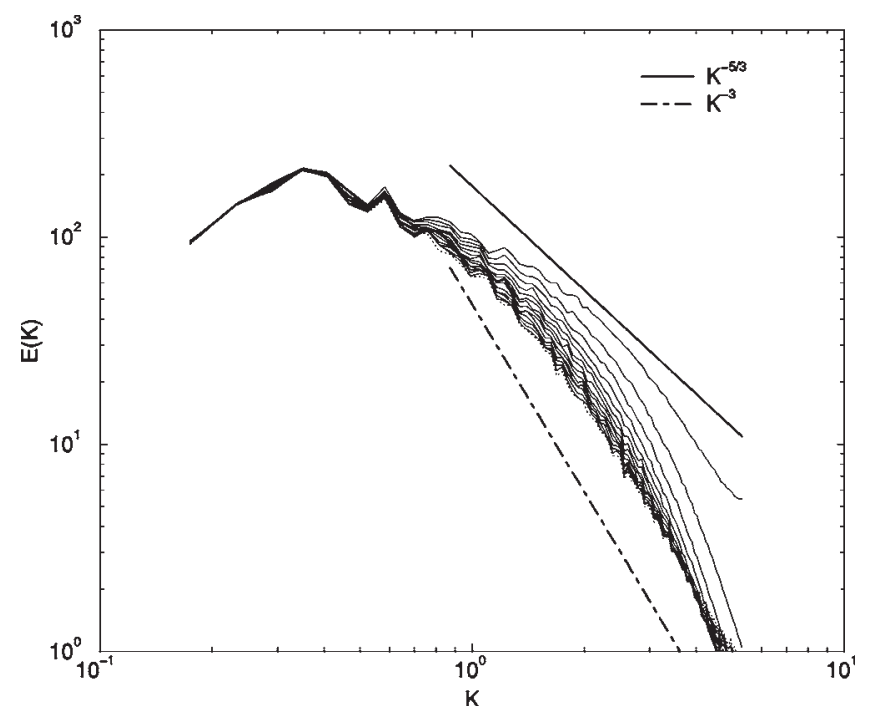

FIG. 10. Time variation of spectrum with a rotation rate of $\Omega=100$ in a $96 \times 96 \times 384$ LES run.

number of 1.82. At the end of the computation, the microRossby number is nearly 0.71 . At the beginning, when rotation is applied, all three models produce a sudden skewness reduction to the level predicted by the scaling law, $S_{k} / S_{k}(t$ $=0) \approx 0.75$. However, the time variations are quite different for the three models. Whereas the micro-Rossby number decreases with time, the AISM remains at nearly the same level. The CZZS model however, shows a small reduction in skewness. Neither follows the scaling law. Only the present model (17) fits the scaling law well; the behavior at the critical micro-Rossby number, $R_{o}^{\omega}=1$, is excellent.

A finer grid with $96 \times 96 \times 384$ nodes is also used to check the influence of the resolution. The initial microRossby number is nearly 2.0. When the computation ends, the final micro-Rossby number is approximately 0.22 . As in Fig. 7, the fit of the Cambon et al. ${ }^{6}$ scaling law is excellent, and also much better than that when a $64 \times 64 \times 256$ grid is used.

Different values of the rotation rate, $\Omega$ $=5,10,20,40,80,160$, covering an initial micro-Rossby number from approximately 4.0 to 0.1 , are then used for different runs on the $64 \times 64 \times 256$ numerical grid. Results are plotted in Fig. 8. The scaling law of Cambon et al. ${ }^{6}$ is verified by the present LES simulation. At very small microRossby numbers, the skewness presents large oscillations. This phenomenon is also present in the study of Cambon et al. ${ }^{6}$

Spectral behavior is also checked on the threedimensional isotropic kinetic energy spectrum $E(K)$. Figures 9 and 10 show the time evolution of $E(K)$ for initial rotation rates of $\Omega=10$ and 100 , respectively. The corresponding initial micro-Rossby numbers are nearly 2.22 and 0.222 . At the end of the computation, the micro-Rossby numbers are about 0.222 and 0.0667 , respectively. Results correspond to a 96 $\times 96 \times 384$ LES runs. Once the rotation is switched on, the spectral slope shifts from nearly $-5 / 3$ to -3 . For the lower rotation rate, an intermediate slope -2 is observed. In the case of smaller micro-Rossby number, it seems that the slope

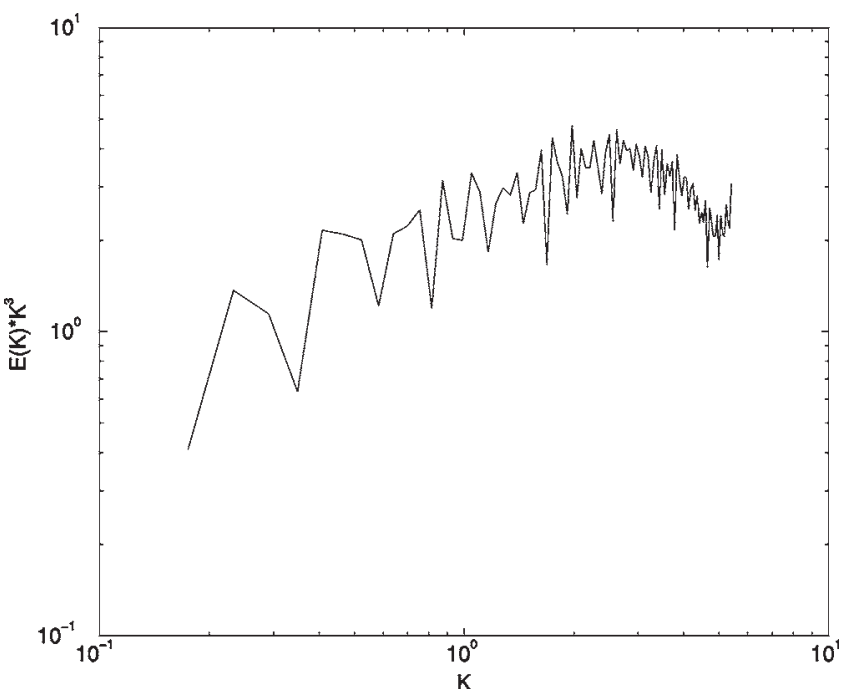

FIG. 11. Compensated spectrum with a rotation rate of $\Omega=10$ at the end of computation in a $96 \times 96 \times 384$ LES run.

is close to -3 . $K^{-3}$ compensated spectra at the end of computation are shown in Figs. 11 and 12, corresponding to the low and the high rotation rates. A plateau seems to exist in both cases, but more obviously for the high rotation rate case. These results are also consistent with the recent study of Yang and Domaradzki. ${ }^{2}$

\section{CONCLUDING REMARKS}

Local volume integrated filtered velocity structure function equations are introduced for subgrid modeling of anisotropic rotating turbulence, and a dynamic subgrid eddy viscosity is proposed. The model is successfully tested in LES of high Reynolds decaying rotating homogeneous turbulence. This model is able to simulate rotating homogeneous turbulence at very high rotation rate (with a micro-Rossby number smaller than 0.1 ). The model can also reproduce the most important feature of the rotating turbulence: the reduc-

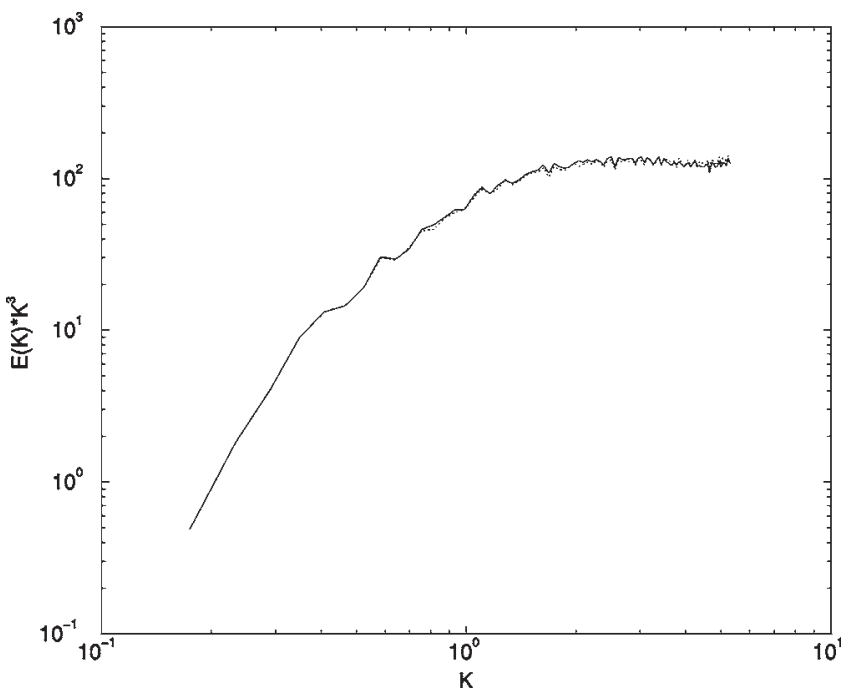

FIG. 12. Compensated spectrum with a rotation rate of $\Omega=100$ at the end of computation in a $96 \times 96 \times 384$ LES run. 
tion of the spectral transfer. A quantitative comparison with the study of Cambon et al. ${ }^{6}$ demonstrates the necessity of the present approach.

\section{ACKNOWLEDGMENTS}

This work was sponsored by the NSFC (Grant 10272065) and by the Sino-French Laboratory LIAMA. Special thanks to the Chun Hui project and the NSFC for supporting the visit of L. Shao at Tsinghua University (Grant 10411150159).

${ }^{1}$ G. X. Cui, H. B.Zhou, Z. S.Zhang, and L. Shao, "A new dynamic subgrid eddy viscosity model with application to turbulent channel flow," Phys. Fluids 16, 2835 (2004).

${ }^{2} \mathrm{X}$. Yang and A. Domaradzki, "Large eddy simulation of rotating decaying," Phys. Fluids 16, 3888 (2004).

${ }^{3}$ C. Cambon, R. Rubinstein, and F. S. Godeferd, "Advances in wave turbulence: Rapidly rotating flows,” New J. Phys. 6, 73 (2004).
${ }^{4}$ G.-H Cottet and A. A. Wray, "Anisotropic grid-based formulas for subgrid-scale models," in Annual Research Brief, Center for Turbulence Research, Stanford University and NASA Ames Research Center (1997).

${ }^{5}$ G.-H Cottet and O. V. Vasilyev, "Comparison of dynamic Smagorinsky and anisotropic subgrid models," in Proceedings of the Summer Program, Center for Turbulence Research (1998).

${ }^{6}$ C. Cambon, N. N. Mansour, and F. S. Godeferd, "Energy transfer in rotating turbulence," J. Fluid Mech. 337, 303 (1997).

${ }^{7}$ R. J. Hill, "Exact second-order structure function relationship," J. Fluid Mech. 468, 317 (2002).

${ }^{8}$ C. M. Casciola, P. Gualtieri, R. Benzi, and R. Piva, "Scale by scale budget and similarity laws for shear turbulence," J. Fluid Mech. 476, 105 (2003).

${ }^{9}$ J. P. Chollet, "Statistical closure to derive a subgrid-scale modelling for large eddy simulations of three dimensional turbulence," Tech. Note TN 206, NCAR (1983)

${ }^{10}$ R. S. Rogallo, "Numerical experiments in homogeneous turbulence," NASA Report, 81315 (1981).

${ }^{11}$ H. Touil, J. P. Bertoglio, and L. Shao, "The decay of turbulence in a bounded domain,” J. Turbul. 3, 1 (2002).

${ }^{12}$ L. Skrbek and S. R. Stalp, "On the decay of homogeneous isotropic turbulence," Phys. Fluids 12, 1997 (2000). 